Pacific Journal of Mathematics

ON THE SEMIGROUP OF BINARY RELATIONS 


\section{ON THE SEMIGROUP OF BINARY RELATIONS}

\section{R. J. Plemmons and M. T. West}

The concepts of row and column bases for an element of $\mathscr{B}_{X}$, the semigroup of binary relations on a set $X$, are introduced by interpreting a binary relation as a boolean matrix; these ideas are then used to characterize the Green's equivalences on $\mathscr{B}_{X}$. It is shown that the class of idempotent relations whose rows and columns form independent sets coincides with the class of partial order relations on subsets of $X$. Regularity in $\mathscr{B}_{x}$ is investigated using these results.

The Green's relations and ideals in the semigroup of binary relations $\mathscr{B}_{X}$ on a set $X$ have been studied primarily in terms of lattice considerations [11], [12]. In this paper we take a more computational approach. By interpreting a relation as a boolean matrix, we introduce the concept of row and column bases and use these ideas to obtain useful characterizations of the Green's relations $\mathscr{L}, \mathscr{R}, \mathscr{L}$ and $\mathscr{D}$ on $\mathscr{B}_{x}$. These results are then used to investigate the ideal structure of $\mathscr{P}_{X}$, in comparison to that of $\mathscr{T}_{X}$, the semigroup of transformations of $X$ into $X$. Some simple tests for regularity of a binary relation are obtained, and by characterizing reduced idempotent relations we show that a regular relation must have the same row rank and column rank.

These results have made possible the determination of the maximal subgroups of $\mathscr{B}_{X}$ [6]. Moreover, the characterization of the Green's relations in terms of binary matrices will hopefully lead to an extension of the combinatorial results given in [4] and [9], in which the numbers of idempotents in the $\mathscr{L}$ and $\mathscr{C}$-classes of $\mathscr{T}_{x}$ are investigated. Other applications may be found in Grapy Theory.

A binary relation on a set $X$ is a subset of $X \times X$, and the set of all binary relations on $X$ is denoted by $\mathscr{B}_{X}$. The product $\alpha \beta$ of two relations $\alpha$ and $\beta$ on $X$ is defined to be the relation

$$
\alpha \beta=\{(a, b) \mid(a, c) \in \alpha \text { and }(c, b) \in \beta \text { for some } c \in X\} \text {. }
$$

The operation is associative and hence $\mathscr{B}_{X}$ is a semigroup. The semigroup $\mathscr{P}_{X}$ of partial transformations on $X$ is a subsemigroup of $\mathscr{B}_{X}$ and it in turn contains $\mathscr{T}_{X}$, the semigroup of transformations on $X$ as a subsemigroup. It was the ideal structure of $\mathscr{T}_{X}$ that motivated many of the ideas in this paper. (See [5] and [1] Vol. I, pp. 51-55.) 
The elements in $\mathscr{B}_{X}$ will usually be denoted by Greek letters. In general, the notation follows [1]. Let $x \in X, H \subseteq X$ and $\alpha \in \mathscr{B}_{X}$. We use the notation:

$$
\begin{aligned}
& \alpha^{-1}=\{(a, b) \mid(b, a) \in \alpha\}, \\
& x \alpha=\{y \in X \mid(x, y) \in \alpha\}, \\
& H \alpha=\{b \mid(h, b) \in \alpha \text { for some } h \in H\}, \alpha H=H \alpha^{-1}, \text { and } \\
& \iota_{H}=\{(h, h) \mid h \in H\} .
\end{aligned}
$$

Also, the universal relation $X \times X$ is denoted by $\omega$, the identity transformation on $X$ by $c$, and the empty relation by $\square$.

Let $\mathscr{B}_{X}$ denote the set of all $X \times X$ matrices $A$ over the boolean algebra $\{0,1\}$, where $a_{x, y}$ denotes the value of $A$ at the point $(x, y)$ of $X \times X$. Then $\mathscr{B}_{n}$ is a semigroup under matrix multiplication. Moreover from [2, Chapter 13], it follows that the map

$$
\phi: \alpha \longrightarrow A, \text { where } a_{x, y}= \begin{cases}1 & \text { if }(x, y) \in \alpha, \\ 0 & \text { otherwise },\end{cases}
$$

is an isomorphism of $\mathscr{B}_{x}$ onto $\mathscr{B}_{n}$. For $x \in X$ we call the set $x \alpha[\alpha x]$ a row [column] of $\alpha$. For example, if $X=\left\{x_{1}, x_{2}, x_{3}\right\}$ and $\alpha=\left\{\left(x_{1}, x_{2}\right),\left(x_{2}, x_{3}\right),\left(x_{3}, x_{1}\right),\left(x_{3}, x_{3}\right)\right\}$ then the relation matrix for $\alpha$ is

$$
\left(\begin{array}{lll}
0 & 1 & 0 \\
0 & 0 & 1 \\
1 & 0 & 1
\end{array}\right),
$$

and the row $x_{3} \alpha$ is the set $\left\{x_{1}, x_{3}\right\}$. These ideas can be extended in the natural way to the case where $X$ is countably infinite. For generality, we shall employ the terms row and column of a relation even when $X$ is not finite.

1. The green's relations on $\mathscr{B}_{x}$. In this section we obtain characterizations of the Green's relations that will be useful in later work.

Two elements $a, b$ of a semigroup $S$ are said to be $\mathscr{L}[\mathscr{R}, \mathscr{T}]$ equivalent if and only if they generate the same principal left [right, two-sided] ideal in $S$. We denote the relation $\mathscr{L} \cap \mathscr{R}$ by $\mathscr{H}$ and the join $\mathscr{L} \vee \mathscr{R}$ of $\mathscr{L}$ and $\mathscr{R}$ by $\mathscr{D}$, that is, $\mathscr{D}$ is the intersection of all the equivalence relations on $S$ that contain $\mathscr{L}$ and $\mathscr{R}$. The equivalence relations $\mathscr{L}, \mathscr{R}, \mathscr{H}$ and $\mathscr{D}$ play an important role in the study of semigroups.

The proof of the following technical lemma is obvious.

Lemma 1.1. Let $\alpha, \beta \in \mathscr{B}_{X}$ and $H \subseteq X$. Then

(1) $H(\alpha \beta)=(H \alpha) \beta$ and $(\alpha \beta) H=\alpha(\beta H)$,

(2) $(\alpha \beta)^{-1}=\beta^{-1} \alpha^{-1}$, 
(3) $\alpha \mathscr{R} \beta$ if and only if $\alpha^{-1} \mathscr{L} \beta^{-1}$,

(4) $\alpha \mathscr{H} \alpha^{-1}$ if and only if $\alpha \mathscr{R} \alpha^{-1}$ or $\alpha \mathscr{L} \alpha^{-1}$, and

(5) $\alpha \mathscr{D} \beta$ if and only if $\alpha^{-1} \mathscr{D} \beta^{-1}$.

Let $\alpha \in \mathscr{B}_{X}$ and $V(\alpha)=\{A \alpha \mid A \subseteq X\}$. Then $V(\alpha)$ is the collection of all unions of rows of $\alpha$ and it forms a lattice under set theoretic inclusion. The following lemma is a combination of results proved by Zaretskii in [12].

Lemma 1.2. Let $\alpha, \beta \in \mathscr{B}_{X}$. Then

(1) $\alpha \mathscr{L} \beta$ if and only if $V(\alpha)=V(\beta)$ and

(2) $\alpha \mathscr{D} \beta$ if and only if $V(\alpha)$ and $V(\beta)$ are lattice isomorphic.

For our purposes it is more convenient to obtain a characterization of the relation $\mathscr{L}$, and dually $\mathscr{R}$, in terms of rows and columns. Using Zaretskii's result we have the following lemma.

Lemma 1.3. Let $\alpha, \beta \in \mathscr{B}_{X}$. Then $\alpha \mathscr{L} \beta[\alpha \mathscr{R} \beta]$ if and only if for each $x \in X$ there exist subsets $H$ and $K$ of $X$ such that $x \alpha=H \beta$ and $x \beta=K \alpha[\alpha x=\beta H$ and $\beta x=\alpha K]$. Moreover $\alpha \mathscr{L} \beta[\alpha \mathscr{R} \beta]$ implies $X \alpha=X \beta[\alpha X=\beta X]$.

Proof. If $\alpha \mathscr{L} \beta$ then there exist relations $\gamma, \delta$ in $\mathscr{B}_{x}$ such that $\gamma \alpha=\beta$ and $\delta \beta=\alpha$. Let $x \in X$, then $x \alpha=(x \delta) \beta$ and $x \beta=(x \gamma) \alpha$ so that $x \alpha=H \beta$ and $x \beta=K \alpha$ for $H=x \delta$ and $K=x \gamma$. The converse is immediate from Lemma 1.2 (1).

Finally, if $\alpha \mathscr{L} \beta$ and $x \in X \alpha$, then there exist $y \in X$ and $H \subseteq X$ such that $x \in y \alpha=H \beta \subseteq X \beta$. Hence $X \alpha \subseteq X \beta$. Similarly $X \beta \subseteq X \alpha$.

For any transformation $\alpha$, the number $|X \alpha|$ is called the rank of $\alpha$ ([1, Ch. 2]). We now introduce the concepts of row and column ranks for relations. These ideas will play an important role in the remainder of the paper. We consider each set mentioned in the following paragraph to be nonempty.

Let $S$ be a set and $\mathscr{A}$ be a collection of nonempty subsets of $S$. Then a subcollection $\mathscr{C}$ of $\mathscr{A}$ is said to be independent if no member of $\mathscr{C}$ is a union of other members of $\mathscr{C}$. The collection $\mathscr{C}$ is said to generate $\mathscr{A}$ if each member of $\mathscr{A}$ is a union of members of $\mathscr{C}$. If $\mathscr{C} \subseteq \mathscr{A}$ is independent and generates $\mathscr{A}$ then $\mathscr{C}$ will be called a basis of $\mathscr{A}$.

Now let $\square \neq \alpha \in \mathscr{B}_{X}$ and $\mathscr{A}=\{x \alpha \mid x \in X, x \alpha \neq \square\}$. If $\mathscr{A}$ has a basis $\mathscr{C}$ then $\mathscr{C}$ is necessarily unique and is called a row basis of $\alpha ;|\mathscr{C}|$ is called the row rank of $\alpha$. Column bases and ranks 
are defined in a dual manner. Of course, if $X$ is finite, then each relation on $X$ has row and column bases. However, for infinite $X$ it is possible to construct relations having no row basis. In particular, if $X$ is the positive integers and $\mathscr{A}$ is the nonempty subsets of $X$ whose complements are finite, then $\mathscr{A}$ has no basis. Thus if we take $\alpha$ to be a relation having as rows the members of $\mathscr{A}$, then $\alpha$ has no row basis.

We say that a relation $\alpha$ is row [column] reduced if the nonempty rows [columns] of $\alpha$ form an independent set. If the relation is both row and column reduced we say it is reduced.

Notice that by Lemma 1.3, a relation $\alpha \in \mathscr{B}_{X}$ has a row basis if and only if $L_{\alpha}$ has row reduced members. In particular if the nonempty rows of $\alpha^{\prime} \in \mathscr{B}_{X}$ form a basis for the rows of $\alpha$ then $\alpha^{\prime} \in L_{\alpha}$. A similar result holds for columns.

The following lemma gives a sufficient condition for the rows of a relation to have a basis. Its proof follows from an application of Zorn's Lemma.

LEMmA 1.4. If $\alpha \in \mathscr{B}_{x}$ has the property that $|x \alpha|<\infty$ for each $x \in X$, then $\alpha$ has a row basis and thus $L_{\alpha}$ contains row reduced members.

The dual of this lemma for column bases also holds. In particular each $\mathscr{D}$-class of $\mathscr{B}_{X}$, when $X$ is finite, contains reduced members.

Notice that our definition of column rank of a relation $\alpha$ was called the rank of $\alpha$ in [1], [5], where $\alpha$ is a transformation. Thus we have a natural extension of the concept of rank to binary relations. Also, there exist relations in $\mathscr{B}_{X},|X| \geqq 4$, for which the row and column ranks are different. For example, the relation $\alpha$ whose matrix is

$$
\left|\begin{array}{llll}
1 & 0 & 1 & 0 \\
0 & 1 & 0 & 1 \\
1 & 1 & 0 & 0 \\
1 & 1 & 1 & 0
\end{array}\right|,
$$

has row rank 3 and column 4. Also, $\alpha$ is column reduced but not row reduced.

Now let $V^{*}(\alpha)=\{x \alpha \mid x \in \alpha X\}$. The next two theorems characterize the Green's relations on reduced elements of $\mathscr{B}_{X}$. All the relations in the remainder of this section are taken to be nonempty.

THEOREM 1.5. Let $\alpha$ and $\beta$ be row reduced relations in $\mathscr{B}_{X}$. Then the following statements are equivalent. 
(A) $\alpha \mathscr{L} \beta$,

(B) $V^{*}(\alpha)=V^{*}(\beta)$, and

(C) $\alpha=\rho \beta$, where $\rho$ is a one-to-one map of $\alpha X$ onto $\beta X$.

Proof. Now the implication (A) implies (B) is immediate from Lemma 1.2, since $V^{*}(\alpha)$ is the basis for $V(\alpha)$.

Assuming $V^{*}(\alpha)=V^{*}(\beta)$, let $\rho$ be the map from $\alpha X$ to $\beta X$ given by $x \rho=y$ if and only if $x \alpha=y \beta$. Then clearly $\rho$ is one-toone from $\alpha X$ onto $\beta X$ and $\alpha=\rho \beta$. Thus (B) implies (C).

Now (C) implies (A), since if $\alpha=\rho \beta$ where $\rho$ is one-to-one from $\alpha X$ onto $\beta X$, then $\rho^{-1} \alpha=\beta$. Then $\alpha$ and $\beta$ generate the same principal left ideal and are thus $\mathscr{L}$-equivalent.

Notice that the since $\alpha \mathscr{R} \beta$ if and only if $\alpha^{-1} \mathscr{L} \beta^{-1}$, Theorem 1.5 has a dual formed by replacing "row" by "column", " $\mathscr{L}$ " by " $\mathscr{R}$ " and " $\rho \beta$ " by " $\beta \rho$ " where $\rho$ is from $X \beta$ onto $X \alpha$. With this in mind we have the following results.

COROLLARY 1.6. Let $\alpha$ and $\beta$ be reduced relations in $\mathscr{B}_{x}$. Then the following statements are equivalent.

(A) $\alpha \mathscr{H} \beta$,

(B) $V^{*}(\alpha)=V^{*}(\beta)$ and $V^{*}\left(\alpha^{-1}\right)=V^{*}\left(\beta^{-1}\right)$, and

(C) $\alpha=\beta \rho=\sigma \beta$ where $\rho$ is a one-to-one map of $X \beta$ onto $X \alpha$ and $\sigma$ is a one-to-one map of $\alpha X$ onto $\beta X$.

LEMma 1.7. If $\alpha \in \mathscr{B}_{X}$ is row [column] reduced, then every member of $R_{\alpha}\left[L_{\alpha}\right]$ is also row [column] reduced. In particular, if $\alpha, \beta \in \mathscr{B}_{X}$ are reduced and $\alpha \mathscr{D} \beta$, then every member of $L_{\alpha} \cap R_{\beta}$ is reduced.

Proof. Let $\alpha$ be row reduced and $\beta \in R_{\alpha}$. We show $\beta$ is row reduced by showing $V^{*}(\beta)=\{x \beta \mid x \in \beta X\}$ is independent. Let $k \in \beta X$ and $\square \neq T \subseteq \beta X$ such that $k \beta=T \beta$. Now by Lemma 1.3, $k \in \beta X=$ $\alpha X$ and $T \subseteq \beta X=\alpha X$. If $\beta \in R_{\alpha}$ then $\alpha=\beta \gamma$ for some $\gamma \in \beta_{X}$. Thus $k \alpha=k \beta \gamma=T \beta \gamma=T \alpha$. Since $\alpha$ is row reduced $V^{*}(\alpha)=\{x \alpha \mid x \in \alpha X\}$ is independent and so $k \in T$. Therefore $V^{*}(\beta)$ is independent, and thus $\beta$ is row reduced.

THEOREM 1.8. Let $\alpha, \beta$ be reduced elements of $\mathscr{B}_{x}$. Then $\alpha \mathscr{D} \beta$ if and only if $\alpha=\sigma \beta \rho$, where $\sigma$ is a one-to-one map of $\alpha X$ onto $\beta X$ and $\rho$ is a one-to-one map of $X \beta$ onto $X \alpha$.

Proof. If $\alpha \mathscr{D} \beta$ then there exists $\gamma \in \mathscr{B}_{X}$ such that $\alpha \mathscr{L} \gamma$ and $\gamma \mathscr{R} \beta$. By Lemma 1.7, $\gamma$ is reduced. By Lemma 1.3, $X \alpha=X \gamma$ and $\gamma X=\beta X$. Now by Theorem 1.5 and its dual, $\alpha=\sigma \gamma$, where $\sigma$ is a 
one-to-one map of $\alpha X$ onto $\gamma X$, and $\gamma=\beta \rho$, where $\rho$ is a one-to-one map of $X \beta$ onto $X \gamma$. Then $\alpha=\sigma \gamma=\sigma \beta \rho$.

Conversely, if $\alpha=\sigma \beta \rho, \sigma, \rho$ as specified We can let $\gamma=\beta \rho$ and note by Theorem 1.5 and its dual, $\alpha \mathscr{L} \gamma$ and $\gamma \mathscr{R} \beta$ so $\alpha \mathscr{D} \beta$.

If $X$ is finite the partial one-to-one mappings of Theorems 1.5 and 1.8 can be taken to be restrictions of permutations of $X$ to the requisite domains. Hence we can restate these results in the following theorem, where $S_{X}$ denotes the symmetric group on $X$.

THEOREM 1.9. Let $\alpha$ and $\beta$ be reduced relations in $\mathscr{B}_{X}, X$ finite. Then

(1) $\alpha \mathscr{L} \beta$ if and only if $\alpha=\rho \beta, \rho \in S_{X}$,

(2) $\alpha \mathscr{R} \beta$ if and only if $\alpha=\beta \sigma, \sigma \in S_{X}$,

(3) $\alpha \mathscr{H} \beta$ if and only if $\alpha=\rho \beta=\beta \sigma, \rho, \sigma \in S_{X}$ and

(4) $\alpha \mathscr{D} \beta$ if and only if $\alpha=\rho \beta \sigma, \rho, \sigma \in S_{X}$.

We show next that a fixed row rank and column rank are associated with each $\mathscr{D}$-class containing reduced relations.

LEMMA 1.10. Let $\alpha$ be a row reduced relation in $\mathscr{B}_{X}$ and suppose $\alpha$ has row rank $r$. Then each member of $L_{\alpha}$ has row rank $r$.

Proof. The proof follows since $r=\left|V^{*}(\alpha)\right|$ and $V^{*}(\alpha)$ is a row basis for each member of $L_{\alpha}$.

TheOREM 1.11. Let $D$ be a $\mathscr{D}$-class of $\mathscr{B}_{X}$ containing reduced relations. Then the members of $D$ have the same row rank $r$ and column rank $c$.

Proof. Let $\alpha$ be a reduced relation in $D$ where $\alpha$ has row rank $r$ and column rank $c$. Then $r=\left|V^{*}(\alpha)\right|$ and $c=\left|V^{*}\left(\alpha^{-1}\right)\right|$ and by Lemma 1.10 and its dual each member of $L_{\alpha}$ has row rank $r$ and each member of $R_{\alpha}$ has column rank $c$. Now let $\beta \in D$. Then there exists $\gamma \in D$ such that $\beta \mathscr{L} \gamma$ and $\gamma \mathscr{R} \alpha$. By Lemma 1.7, each member of $R_{\alpha}$ is row reduced so $\gamma$ has a row basis and has row rank $r$ since $\gamma X=\alpha X$. Thus $\beta$ has row rank $r$ since $\beta \mathscr{L} \gamma$. Also there exists $\delta \in D$ such that $\beta \mathscr{R} \delta$ and $\delta \mathscr{L} \alpha$. Then $\delta$ is column reduced with column rank $c$ and so $\beta$ has column rank $c$, by Lemmas 1.7 and 1.10.

Notice that elements having the same row rank and some column rank need not be $\mathscr{D}$-equivalent. For example, $\omega \mid c=\{(x, y) \in X \times X \mid$ $x \neq y\}$ and $\iota$ have the same $\operatorname{rank}|X|$ but are not in the same $\mathscr{D}$-class.

We conclude this section with some remarks concerning ideals in $\mathscr{B}_{X}, X$ finite. 
Let $|X|=n>2$ and let $\mathscr{T}_{X}$ denote the full transformation semigroup on $X$. Notice that each $\alpha \in \mathscr{T}_{X}$ is column reduced. The column rank of $\alpha$ is $|X \alpha|$ as defined in [1, vol. I, p. 52]. Now $\alpha$ is row reduced if and only if it is a permutation.

The ideal structure of $\mathscr{T}_{X}$ is quite simple. Each ideal is principle and the ideals form a principal series [1, vol. I, pp. 74-75]. Also $\mathscr{T}_{X}$ is semisimple since $I^{2}=I$ for each ideal $I$. In fact, basically the same ideal structure holds for $\mathscr{P}_{X}$, the semigroup of partial transformations on $X$. However, the ideal structure of $\mathscr{B}_{X}$ is not quite so simple. For example, consider the principal ideals $J(\omega \backslash \ell)=$ $\mathscr{B}_{X}(\omega \backslash \iota) \mathscr{B}_{X}$ and $J(\alpha)=\mathscr{B}_{X} \alpha \mathscr{B}_{X}$ where $\alpha=\iota \cup\{(x, y)\}$ and $x \neq y$. Then the only elements in $J(\omega \mid c)$ having rank $n$ are those in the $\mathscr{D}$-class containing $\omega \backslash \ell,\left\{\omega \backslash \rho \mid \rho \in S_{X}\right\}$. Thus $\alpha \notin J(\omega \backslash \ell)$ and similarly $\omega \backslash \ell \notin J(\alpha)$. Thus the ideal $I=J(\omega \backslash \ell) \cup J(\alpha)$ is not principal. Since $J(\omega \backslash c) \nsubseteq J(\alpha)$ and $J(\alpha) \nsubseteq J(\omega \backslash c)$, the principal ideals of $\mathscr{B}_{X}$ do not form a chain. In fact, they do not even form a lattice. Moreover, since $[J(\omega \backslash \iota)]^{2} \neq$ $J(\omega \backslash \iota), \mathscr{B}_{X}$ is not semisimple when $|X|>2$.

Finally, another major difference in the structures of $\mathscr{B}_{x}$ and $\mathscr{T}_{X}$ is that each maximal subgroup of $\mathscr{T}_{X}$ [and of $\mathscr{P}_{X}$ ] is a symmetric group, whereas the class of maximal subgroups of semigroups $\mathscr{B}_{X}$ of binary relations includes all finite groups [6].

2. Regularity in $\mathscr{B}_{X}$. An element $a$ in a semigroup $T$ is said to be a regular element if and only if $a \in a T a$; otherwise, $a$ is called irregular. It has been shown that in any $\mathscr{D}$-class $D$ of $T$, either every element is regular or else every element in $D$ is irregular. If the elements of $D$ are regular then $D$ is called a regular $\mathscr{D}$-class. Moreover $D$ is regular if and only if every $\mathscr{L}$ and $\mathscr{R}$ class of $T$ in $D$ contains an idempotent element [1, vol. I, p. 58].

Regularity in $\mathscr{B}_{X}$ was first investigated by $\mathrm{K}$. Zaretskii in [11] and [12]. He showed that a relation $\alpha \in \mathscr{B}_{X}$ is regular if and only if the collection of sets $V(\alpha)=\{A \alpha \mid A \subseteq X\}$ forms a completely distributive lattice under inclusion. J. Yang has a shorter proof of this result in [10]. However, these characterizations can be rather difficult to use. In this section we relate regularity to row and column rank and show that if $\alpha$ is regular then its row rank and its column rank must be the same. However, this condition is not necessary as we shall show. We also consider idempotent relations and derive a useful test for regularity.

We first relate the ranks of products of relations to the ranks of the factors.

LEMma 2.1. Let $\alpha, \beta \in \mathscr{B}_{X}$ and suppose $\alpha$ and $\beta$ have row and column bases respectively. Let $r$ be the row rank of $\alpha$ and $c$ be the 
column rank of $\beta$. Then if $\alpha \beta$ has row and column bases, the row rank of $\alpha \beta \leqq r$, and the column rank of $\alpha \beta \leqq c$.

Proof. Now $\alpha \mathscr{L} \alpha^{\prime}$ and $\beta \mathscr{R} \beta^{\prime}$ where $\alpha^{\prime}$ is row reduced and $\beta^{\prime}$ is column reduced. Then since $\mathscr{L}$ is a right congruence and $\mathscr{R}$ is a left congruence on $\mathscr{B}_{X}, \alpha \beta \mathscr{D} \alpha^{\prime} \beta^{\prime}$ so that $\alpha \beta$ and $\alpha^{\prime} \beta^{\prime}$ have the same row and column ranks by Theorem 1.11. Moreover $r=\left|\alpha^{\prime} X\right|$ and $c=\left|X \beta^{\prime}\right|$. Then row rank $\alpha \beta=$ row rank $\alpha^{\prime} \beta^{\prime} \leqq\left|\alpha^{\prime} \beta^{\prime} X\right| \leqq$ $\left|\alpha^{\prime} X\right|=r$ and column $\operatorname{rank} \alpha \beta=$ column $\operatorname{rank} \alpha^{\prime} \beta^{\prime} \leqq\left|X \alpha^{\prime} \beta^{\prime}\right| \leqq$ $\left|X \beta^{\prime}\right|=c$.

It is possible, however, for the row [column] rank of the product to be greater than the row [column] rank of the last [first] factor.

Now if $Y \subseteq X$ then the map $\phi$ from $\mathscr{B}_{Y}$ into $\mathscr{B}_{X}$ given by $\alpha \phi=$ $\{(a, b) \in X \times X \mid(a, b) \in \alpha\}$ is an isomorphism. Thus we have the following lemma.

Lemma 2.2. Let $X$ and $Y$ be sets where $|Y| \leqq|X|$. Then there is an isomorphism of $\mathscr{B}_{Y}$ into $\mathscr{B}_{X}$.

An element $\alpha \in \mathscr{B}_{x}$ is regular if and only if the $\mathscr{D}$-class $D$ containing $\alpha$ is regular. But $D$ is regular if and only if it contains an idempotent. Thus it is important to investigate idempotent relations.

Lemma 2.3. Let $D$ be a regular $\mathscr{D}$-class of $\mathscr{B}_{X}$ and assume $D$ contains reduced elements. Then $D$ contains a reduced idempotent.

Proof. Using Theorem 1.8, we see that $D$ must contain a reduced relation $\alpha$ where either $X \alpha \subseteq \alpha X$ or $\alpha X \subseteq X \alpha$. Suppose $X \alpha \subseteq \alpha X$ and let $\alpha X=Y$. A dual argument holds when $\alpha X \subseteq X \alpha$. Then since $|Y| \leqq|X|$, there is an isomorphism $\phi$ of $\mathscr{B}_{Y}$ into $\mathscr{B}_{X}$. Let $\alpha^{\prime}$ be the pre-image of $\alpha$ under $\phi$. Then $\alpha^{\prime}$ is row and column reduced and has no empty rows. Then each element in the $\mathscr{L}$-class $L^{\prime}$ of $\mathscr{B}_{Y}$ containing $\alpha^{\prime}$ is row and column reduced. But since $\alpha$ is regular so is $\alpha^{\prime}$, and thus $L^{\prime}$ contains an idempotent $\varepsilon^{\prime}$. Then $\varepsilon=$ $\varepsilon^{\prime} \phi$ is a reduced idempotent in $D$.

We now characterize the class of reduced idempotents. By a partial order relation in $\mathscr{B}_{X}$ we mean a relation $\alpha$ such that for some subset $Y$ of $X, \alpha \subseteq Y \times Y$ and $\alpha$ is a partial order relation on $Y$. That is, $\alpha \cap \alpha^{-1}=\iota_{Y}$ and $\alpha$ is transitive.

THEOREM 2.4. The class of reduced idempotents in $\mathscr{B}_{X}$ coincides with the class of partial order relations on subsets of $X$. 
Proof. If $\alpha$ is a reduced idempotent then $(x \alpha) \alpha=x \alpha$ implies $x \in X \alpha$ for all $x \in \alpha X$. Then $\alpha X \subseteq X \alpha$, and similarly $\alpha(\alpha x)=\alpha x$ implies $X \alpha \leqq \alpha X$. Let $Y=X \alpha=\alpha X$. Then $x \in x \alpha$ for all $x \in Y$ implies $\iota_{Y} \subseteq \alpha$ so $\alpha$ is reflexive on $Y$. Now if $(x, y) \in \alpha$ where $x \neq y$, then since $x \alpha \neq y \alpha$ there exists $z \in X$ such that either $(x, z) \in \alpha$ and $(y, z) \notin \alpha$ or $(x, z) \notin \alpha$ and $(y, z) \in \alpha$. But this would be impossible if $(y, x) \in \alpha$ since an idempotent relation is always transitive. Thus $\alpha$ is antisymmetric and is therefore a partial order relation on $Y$.

Conversely, suppose $\alpha$ is a partial order relation on a subset $Y$ of $X$. Then $\alpha^{2}=\alpha$ since it is reflexive and transitive on $Y$. Now if $x \alpha=H \alpha$ for some $x \in Y$ and $H \subseteq Y$, then $x \in h \alpha$ for some $h \in H$. But $h \in x \alpha$ implies that $(x, h)$ and $(h, x) \in \alpha$. Thus $x=h$ and so $\alpha$ is row reduced. Similarly, $\alpha$ is column reduced.

We show next that if a regular relation has row and column bases, then its row and column rank must be equal.

THeOREM 2.5. Let $D$ be a regular $\mathscr{D}$-class containing reduced relations and suppose the elements in $D$ have row rank $r$ and column rank $c$. Then $r=c$.

Proof. By Lemma 2.3 and Theorem 2.4, $D$ contains a reduced idempotent $\alpha$ where $X \alpha=\alpha X=Y$. Now since $\alpha$ is reduced, $|\alpha X|=r$ and $|X \alpha|=c$. Thus $r=c$.

COROLlaRY 2.6. Let $\alpha$ be a relation in $\mathscr{B}_{X}$ having row rank $r$ and column rank $c$. Then $\alpha$ is regular only if $r=c$.

We point out that the converse of Corollary 2.6 does not hold. In particular, $\omega \mid c$ is irregular for $|X|>2$ and it has row rank= $|X|=$ column rank. The fact that $\omega \mid c$ is irregular for $|X|>2$ will follow from Corollary 2.10.

THEOREM 2.7. Each regular $\mathscr{D}$-class of $\mathscr{B}_{X}$, X finite, containing reduced relations contains an idempotent $\alpha$ whose relation matrix $A$ is in lower triangular form, that is, where $A$ has the form

$$
A=\left(\begin{array}{ccccccc|c}
1 & 0 & 0 & \cdot & \cdot & \cdot & 0 & \\
* & 1 & 0 & \cdot & \cdot & \cdot & 0 & \\
* & * & 1 & \cdot & \cdot & \cdot & 0 & 0 \\
\cdot & \cdot & \cdot & & & & \cdot & \\
\cdot & \cdot & \cdot & & & & \cdot \\
\cdot & \cdot & \cdot & & & & \cdot \\
* & * & * & \cdot & \cdot & \cdot & 1_{r} \\
\hline & & 0 & & & & 0
\end{array}\right)
$$


where $r$ is the row and column rank of $\alpha$.

Proof. By Theorem 2.4, $D$ contains a partial order relation $\beta$ on a subset $Y$ of $X$. Then $r=|Y|$ is the rank of the members of $D$, and since $\beta$ is a partial order on $Y$, there is a permutation $\rho$ of $Y$ such that the relation matrix for the idempotent $\alpha=\rho \beta \rho^{-1}$ is in lower triangular form. The fact that $\alpha \in D$ follows from Theorem 1.8.

We now obtain a simple criterion for a reduced relation to be regular. It becomes quite useful in case $X$ is finite.

THEOREM 2.8. Let $\alpha$ be a reduced relation in $\mathscr{B}_{x}$. Then $\alpha$ is regular if and only if there exists a one-to-one partial transformation $\rho$ of $X$, such that $\alpha=\alpha \rho \alpha$.

Proof. If $\alpha$ is regular then the $\mathscr{D}$-class containing contains a reduced idempotent $\varepsilon$ by Lemma 2.3. Then by Theorem 1.8, there exist one-to-one partial transformations $\delta$ and $\sigma$ of $X$ such that $\varepsilon=$ $\delta \alpha \sigma$. Then $\delta \alpha \sigma=\delta \alpha \sigma \delta \alpha \sigma$ so that $\alpha=\alpha(\sigma \delta) \alpha$. Letting $\sigma \delta=\rho$ we have our result.

Since each regular $\mathscr{D}$-class of $\mathscr{B}_{X}, X$ finite, contains reduced relations, the following corollary gives a useful criterion for determining if a $\mathscr{D}$-class is regular.

CoRollary 2.9. Let $X$ be finite. Then a reduced relation $\alpha \in \mathscr{B}_{X}$ is regular if and only if $\alpha=\alpha \rho \alpha$ for some $\rho \in S_{X}$.

Finally, the following corollary identifies another class of irregular relations.

COROLLARY 2.10. If $\alpha \neq \square$ is a reduced relation with the property that $x \alpha \leqq y \alpha$ implies $x=y$ for all $x, y \in \alpha X$, then $\alpha$ is either a one-to-one partial transformation of $X$ or is irregular.

Proof. Suppose $\alpha$ is regular. Then by the theorem $\alpha=\alpha \rho \alpha$ where $\rho$ is a one-to-one map from $X \alpha$ onto $\alpha X$. Now $y \alpha=y(\alpha \rho \alpha)$ for all $y \in \alpha X$, so that if $x \in y(\alpha \rho)$ then $x \alpha \cong y \alpha$, whence $x=y$. Thus $y(\alpha \rho)=\{y\}$ and therefore $\alpha \rho=\iota_{\alpha X}$. This implies $\alpha=\rho^{-1}$, so the corollary is proved.

As an illustration of this corollary we see that the relation with matrix 


$$
\left(\begin{array}{llll}
1 & 0 & 1 & 0 \\
0 & 1 & 0 & 1 \\
1 & 0 & 0 & 1 \\
0 & 1 & 1 & 0
\end{array}\right)
$$

is necessarily irregular.

The authors wish to thank the referee for his helpful comments. We also wish to thank Professor Mario Petrich for pointing out that, in general, $\mathscr{J} \neq \mathscr{D}$ on $\mathscr{B}_{X}$ and for pointing to several papers on the subject by Russian authors.

\section{REFERENCES}

1. A. H. Clifford and G. B. Preston, The Algebraic Theory of Semigroups, Amer. Math. Soc. Surveys, vols. I and II, Providence, 1961 and 1967.

2. G. Birkhoff, Lattice Theory, A.M.S. Colloquium Publ., vol. 25, 2nd ed., Providence, 1948.

3. J. A. Green, On the structure of semigroups, Ann. of Math. 54 (1951), 163-172.

4. B. Harris and L. Schoenfeld, The number of idempotent elements in symmetric semigroups, J. Combinatorial Theory 3 (1967), 122-135.

5. A. I. Malcev, Symmetric groupoids, Mat. Sbornik 31 (1952), 136-151.

6. S. J. Montague and R. J. Plemmons, Maximal subgroups of the semigroup of relations, J. of Algebra 13 (1969), 575-588.

7. B. M. Schein, Semigroups of transformations, presented at International Conference on Semigroups, Bratislava, Czechoslovakia, June 1968.

8. S. Schwarz, The semigroup of binary relations on a finite set, presented at International Conference on Semigroups, Bratislava, Czechoslovakia, June 1968 (to appear in Czech. J. Math.)

9. M. Tainter, $A$ characterization of idempotents in semigroups, J. Combinatorial Theory 5 (1968), 370-373.

10. Jaw-Ching Yang, $A$ theorem on the semigroup of binary relations, Proc. Amer. Math. Soc. 22 (1969), 134-135.

11. K. A. Zaretskii, Regular elements in the semigroup of binary relations, Uspehi Mat. Nauk. (17) 3 (1962), 105-108.

12. - The semigroup of binary relations, Mat. Sbornik 61 (1963), 291-305.

Received January 5, 1970. This research was done under National Science Foundation Grant GP-8696.

The University of Tennessee 



\section{PACIFIC JOURNAL OF MATHEMATICS}

\section{EDITORS}

\author{
H. SAMELSON \\ Stanford University \\ Stanford, California 94305 \\ Richard Pierce \\ University of Washington \\ Seattle, Washington 98105
}

\author{
J. DugundJI \\ Department of Mathematics \\ University of Southern California \\ Los Angeles, California 90007 \\ RICHARD ARENS \\ University of California \\ Los Angeles, California 9.0024
}

\section{ASSOCIATE EDITORS}

\begin{tabular}{|c|c|}
\hline E. F. BECKENBACH & K. YoshidA \\
\hline \multicolumn{2}{|c|}{ SUPPORTING INSTITUTIONS } \\
\hline UNIVERSITY OF BRITISH COLUMBIA & STANFORD UNIVERSITY \\
\hline CALIFORNIA INSTITUTE OF TECHNOLOGY & UNIVERSITY OF TOKYO \\
\hline UNIVERSITY OF CALIFORNIA & UNIVERSITY OF UTAH \\
\hline MONTANA STATE UNIVERSITY & WASHINGTON STATE UNIVERSITY \\
\hline UNIVERSITY OF NEVADA & UNIVERSITY OF WASHINGTON \\
\hline NEW MEXICO STATE UNIVERSITY & $* \quad * \quad *$ \\
\hline OREGON STATE UNIVERSITY & AMERICAN MATHEMATICAL SOCIETY \\
\hline UNIVERSITY OF OREGON & CHEVRON RESEARCH CORPORATION \\
\hline OSAKA UNIVERSITY & TRW SYSTEMS \\
\hline UNIVERSITY OF SOUTHERN CALIFORNIA & NAVAL WEAPONS CENTER \\
\hline
\end{tabular}

The Supporting Institutions listed above contribute to the cost of publication of this Journal, but they are not owners or publishers and have no responsibility for its content or policies.

Mathematical papers intended for publication in the Pacific Journal of Mathematics should be in typed form or offset-reproduced, (not dittoed), double spaced with large margins. Underline Greek letters in red, German in green, and script in blue. The first paragraph or two must be capable of being used separately as a synopsis of the entire paper. The editorial "we" must not be used in the synopsis, and items of the bibliography should not be cited there unless absolutely necessary, in which case they must be identified by author and Journal, rather than by item number. Manuscripts, in duplicate if possible, may be sent to any one of the four editors. Please classify according to the scheme of Math. Rev. Index to Vol. 39. All other communications to the editors should be addressed to the managing editor, Richard Arens, University of California, Los Angeles, California, 90024.

50 reprints are provided free for each article; additional copies may be obtained at cost in multiples of 50 .

The Pacific Journal of Mathematics is published monthly. Effective with Volume 16 the price per volume (3 numbers) is $\$ 8.00$; single issues, $\$ 3.00$. Special price for current issues to individual faculty members of supporting institutions and to individual members of the American Mathematical Society: $\$ 4.00$ per volume; single issues $\$ 1.50$. Back numbers are available.

Subscriptions, orders for back numbers, and changes of address should be sent to Pacific Journal of Mathematics, 103 Highland Boulevard, Berkeley, California, 94708.

PUBLISHED BY PACIFIC JOURNAL OF MATHEMATICS, A NON-PROFIT CORPORATION

Printed at Kokusai Bunken Insatsusha (Internatıonal Academic Printing Co., Ltd.), 7-17, Fujimi 2-chome, Chiyoda-ku, Tokyo, Japan. 


\section{Pacific Journal of Mathematics}

\section{Vol. 35, No. $3 \quad$ November, 1970}

John D. Arrison and Michael Rich, On nearly commutative degree one algebras . . . 533

Bruce Alan Barnes, Algebras with minimal left ideals which are Hilbert spaces . . . . 537

Robert F. Brown, An elementary proof of the uniqueness of the fixed point index . . . 549

Ronn L. Carpenter, Principal ideals in F-algebras .................... 559

Chen Chung Chang and Yiannis (John) Nicolas Moschovakis, The Suslin-Kleene

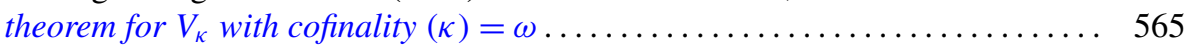

Theodore Seio Chihara, The derived set of the spectrum of a distribution

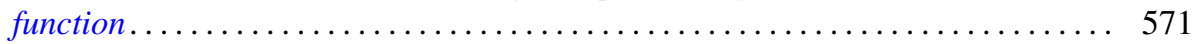

Tae Geun Cho, On the Choquet boundary for a nonclosed subspace of $C(S) \ldots \ldots \quad 575$

Richard Brian Darst, The Lebesgue decomposition, Radon-Nikodym derivative,

conditional expectation, and martingale convergence for lattices of sets .......

David E. Fields, Dimension theory in power series rings . . . . . . . . . . . .

Michael Lawrence Fredman, Congruence formulas obtained by counting

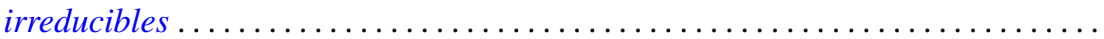

John Eric Gilbert, On the ideal structure of some algebras of analytic functions.....

G. Goss and Giovanni Viglino, Some topological properties weaker than

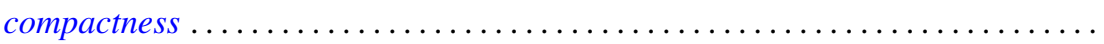

581

601

625

George Grätzer and J. Sichler, On the endomorphism semigroup (and category) of

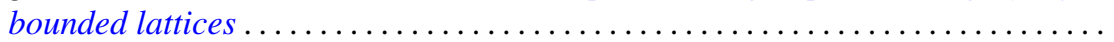

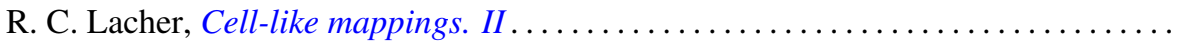

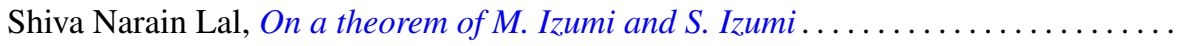

661

Howard Barrow Lambert, Differential mappings on a vector space ...............

Richard G. Levin and Takayuki Tamura, Notes on commutative power joined

semigroups.

Robert Edward Lewand and Kevin Mor McCrimmon, Macdonald's theorem for quadratic Jordan algebras.

J. A. Marti, On some types of completeness in topological vector spaces ....

Walter J. Meyer, Characterization of the Steiner point

717

Saad H. Mohamed, Rings whose homomorphic images are $q$-rings ...

727

Thomas V. O'Brien and William Lawrence Reddy, Each compact orientable surface

of positive genus admits an expansive homeomorphism ...

737

Robert James Plemmons and M. T. West, On the semigroup of binary relations...

743

Calvin R. Putnam, Unbounded inverses of hyponormal operator . .

755

William T. Reid, Some remarks on special disconjugacy criteria for differential

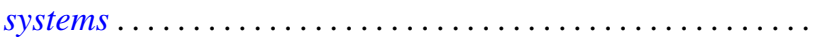

C. Ambrose Rogers, The convex generation of convex Borel sets in euclidean

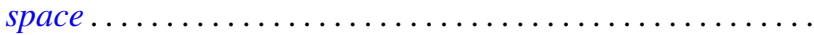

S. Saran, A general theorem for bilinear generating functions .

S. W. Smith, Cone relationships of biorthogonal systems ......

Wolmer Vasconcelos, On commutative endomorphism rings ....

795

Vernon Emil Zander, Products of finitely additive set functions from Orlicz

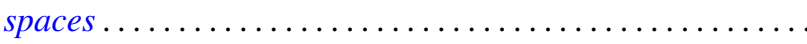

G. Sankaranarayanan and C. Suyambulingom, Correction to: "Some renewal

theorems concerning a sequence of correlated random variables" .

Joseph Zaks, Correction to: "Trivially extending decompositions of $E^{n}$ ”....... 805

Dong Hoon Lee, Correction to: "The adjoint group of Lie groups" ............ 805

James Edward Ward, Correction to: "Two-groups and Jordan algebras". 